\title{
Kinematics and Workspace Atlas of 3-PSS/7R Hybrid Redundantly Driven Mechanism
}

\author{
Qisheng ZHANG*, Ruiqin LI**, Jingjing LIANG*** \\ *School of Mechanical Engineering, North University of China, Taiyuan 030051, China, \\ E-mail: zhangqisheng_qs@nuc.edu.cn \\ **School of Mechanical Engineering, North University of China, Taiyuan 030051, China, E-mail: liruiqin@nuc.edu.cn \\ (Corresponding author) \\ ***School of Mechanical Engineering, North University of China, Taiyuan 030051, China \\ cross ref http://dx.doi.org/10.5755/j02.mech.28987
}

\section{Introduction}

The concept of hybrid-driven mechanism was first proposed by Tokuz [1]. This type of mechanism with twodegrees of freedom is controlled by a constant velocity (CV for short) motor (uncontrolled) and a servo motor (real-time control). The CV motor provides main power and movement required. The servo motor provides a flexible movement regulation by controlling the output of the servo motor of limited power capacity. Therefore, hybrid-driven mechanism takes advantage of the complementary characteristics of both motors to generate a programmable range of highly nonlinear output motions with high power capacities [2]. Hybrid-driven mechanism is capable of generating precise and flexible motion and is suitable for applications that only require machines with certain flexibility and intelligence to complete similar tasks, such as medical rehabilitation, packaging engineering and automatic production line.

Up to date, a series of theoretical results have been reported about kinematics [3-6], dynamics [7 - 8], application research [9-11], control [12 - 13] and optimization design [14 - 15], etc. on hybrid driven planar five-bar mechanism, seven-bar mechanism. Zi and Sun et al. [16 - 19] studied kinematics and dynamics on hybrid-driven based cable parallel manipulator with 3 6 limbs, including singularity, workspace, and application, etc. Each limb consists of cable and closed chain five-bar mechanism.

The traditional $n$-DOF hybrid driven mechanism can be regarded as composed of $n$ mutually coupled limbs, which has $n$ motion inputs as $\boldsymbol{\theta}=\left(\theta_{1}, \theta_{2}, \cdots, \theta_{n}\right)^{\mathrm{T}}$, $3 \leq n \leq 6$ and the trajectory generalized coordinates of the moving platform outputs as $\boldsymbol{X}=\left(x_{1}, x_{2}, \cdots, x_{n}\right)^{\mathrm{T}}$, $3 \leq n \leq 6$. The $n$ independent position equations $f(\boldsymbol{\theta})=g(\boldsymbol{X})$ are established using closed vector method. The derivative of the equation of position with respect to time gives the Jacobian of velocity. Because the velocity of the CV motor is constant, the full rank $n$-DOF $(3 \leq n \leq 6)$.

Jacobian matrix of hybrid driven mechanism does not exist, thus the mechanism is not controllable. The redundantly driven mode is formed, when one or several limbs are added to the mechanism and the driver is added at the same time. The velocity Jacobian matrix is non-full rank, and there are countless inverse kinematics solutions. Extending the configuration of hybrid-driven mechanism from plane field to space field is a starting point of this paper.

Zhang et al. [20, 21] introduced a family of parallel mechanisms with 3, 4 and 5 degrees of freedom with passive limb. These mechanisms are that a passive limb fully provides the freedom of the parallel mechanism and only provides all the constraints that the moving platform requires, and then the number of active chains is equal to the number of degree of freedoms of the parallel mechanism, and the active limb provides only the driver for the moving platform. When a driver is added to the passive limb, a redundant parallel mechanism is formed [22 - 24]. Redundantly driven parallel mechanism is a type of parallel mechanism, in which the number of input links is more than the degrees of freedom of the mechanism.

At present, the general redundantly driven parallel mechanism is mainly as: (1) Directly making the passive joint of the non-redundant parallel mechanism into the actuated joint. Zhou et al. [25] researched the configuration of 5-UPS/PRPU parallel machine tool; (2) Adding driving limb in non-redundant parallel mechanism by using self-averaging redundant driving interface module. Liu et al. [26] designed an improved 3-degrees-of-freedom (DOF) redundantly actuated parallel platform 2UPS-RPS-PS which consists of four actuations. The common characteristics of the mechanism is derived from the Stewart platform, add a limb connect to static platform and moving platform with driving or without driving. Some limbs are only constraint the freedom of the whole mechanism. The limb has a positive effect on the overall performance of the parallel mechanism.

Compared with the general parallel mechanism, it has the advantages of high rigidity, excellent operation performance, bearing capacity and high positioning accuracy [27 - 29]. The research of spatial connecting rod type hybrid-driven mechanism is seldom reported in literature, which greatly limits the application field of hybrid-driven mechanism.

In this paper, a novel 3-DOF 3-PSS/7R hybrid redundantly driven mechanism (HRDM for short) is proposed for spatial motion. The 3-DOF HRDM includes three PSS driving limbs and one $7 \mathrm{R}$ middle limb. The middle limb is composed of a planar 5-bar mechanism and a spatial linkage mechanism in series, which is driven by $\mathrm{CV}$ motor and servo motor together. The mechanism has 3 degrees of freedom, but 5 drives. Therefore, the drives of the mechanism are redundant. The middle limb is the redundant limb with hybrid driven, which determines the freedom of the HRDM, which is called hybrid redundantly driven limb (HRDL for short). 


\section{Kinematic Modelling of the 3-PSS/7R HRDM}

Fig. 1 shows the schematic diagram of the 3PSS/7R HRDM. The 3-PSS/7R parallel mechanism is comprised of a base, a moving platform, three identical PSS limbs, and one middle limb. One end of the PSS limb connects the base with the prismatic pair $\mathrm{P}$, and the other end connects the moving platform with the spherical pair S. In the initial position, three prismatic drives are distributed symmetrically on the circumference of the base.

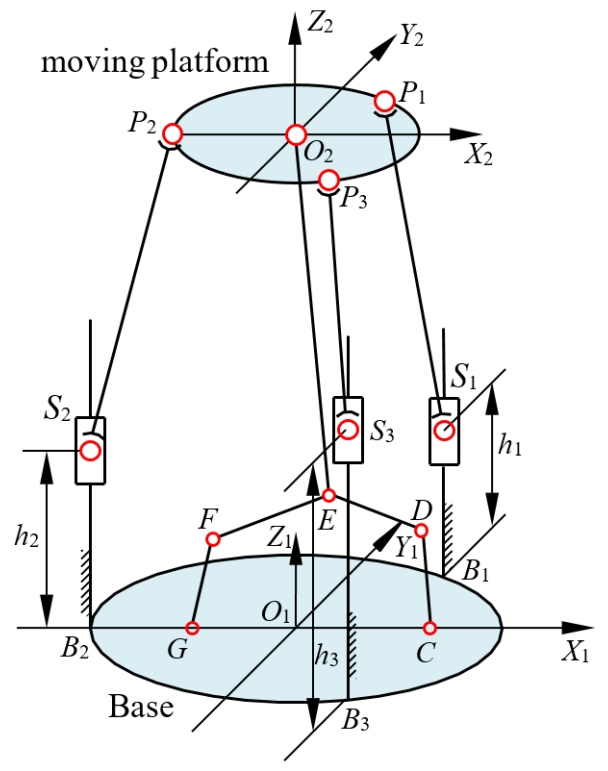

Fig. 1 The schematic diagram of the 3-PSS/7R HRDM

The middle limb is composed of a planar five-bar mechanism $C D E F G$ and a connecting link $E O_{2}$ in series. The frame of the planar five-bar mechanism $C D E F G$ is fixed in the base, which is driven by a $\mathrm{CV}$ motor and a servo motor. The two ends of the connecting link connect the reference point $\mathrm{O}_{2}$ of the moving platform and point $E$ of the five-bar mechanism with revolute joints, respectively. The moving platform of the 3-PSS/7R mechanism can realize 2T1R (two translations and one rotation) movements within the workspace range by changing the lengths of the three driving links and changing the driving angle of the servo motor.

In Fig. 1, the radii of the base and the moving platform are marked as $R, r$, respectively. The three guide paths $S_{i}(i=1,2,3)$ of the prismatic pairs are symmetrically distributed on the circumference of the base. The three spherical pairs $P_{i}(i=1,2,3)$ are symmetrically distributed on the circumference of the moving platform.

Figure 1 shows two coordinate systems. The base coordinate system $O_{1}-X_{1} Y_{1} Z_{1}$, marked as $\left\{O_{1}\right\}$, is connected to the base. The coordinate origin $O_{1}$ is located at the centre of the base. $X_{1}$ axis passes through point $B_{2}$ and points to the right, while points $B_{1}$ and $B_{3}$ are symmetric about $X_{1}$ axis. $Z_{1}$ axis is perpendicular to the base and the direction is upward.

The moving coordinate system $\mathrm{O}_{2}-\mathrm{X}_{2} \mathrm{Y}_{2} \mathrm{Z}_{2}$, marked as $\left\{\mathrm{O}_{2}\right\}$, is connected to the moving platform. The coordinate origin $\mathrm{O}_{2}$ is located at the centre of the moving platform. $X_{2}$ axis passes through point $P_{2}$ and points to the right, while points $P_{1}$ and $P_{3}$ are symmetric about $X_{2}$ axis. $Z_{2}$ axis is perpendicular to the moving platform and point away from the mechanism.

The frame $C G$ of the five-bar mechanism coincides with $X_{1}$ axis in the $\left\{O_{1}\right\}$. The axes of the revolute pairs $C$, $D, F, G$ are parallel to $Y_{1}$ axis. In the initial position, the direction of $Z_{1}$ axis coincides with that of $Z_{2}$ axis. $X_{1}$ axis is parallel to $X_{2}$ axis. $Y_{1}$ axis is parallel to $Y_{2}$ axis.

In the planar five-bar mechanism $C D E F G$, the $\mathrm{CV}$ motor drives link $C D$ to move, the servo motor drives link $G F$ to move. The output is the movement trajectory of point $E$. The lengths of the planar five-bar mechanism are marked as $|C D|=l_{1},|D E|=l_{2},|E F|=l_{3},|F G|=l_{4},\left|C O_{1}\right|=\left|G O_{1}\right|=$ $=l_{5},\left|E O_{2}\right|=l_{6}$, respectively. The rotation angle of link $l_{i}$ are marked as $q_{i}(i=1,2,3,4)$, where $q_{i}=\theta_{i},(i=1,2)$, as shown in Fig. 2.

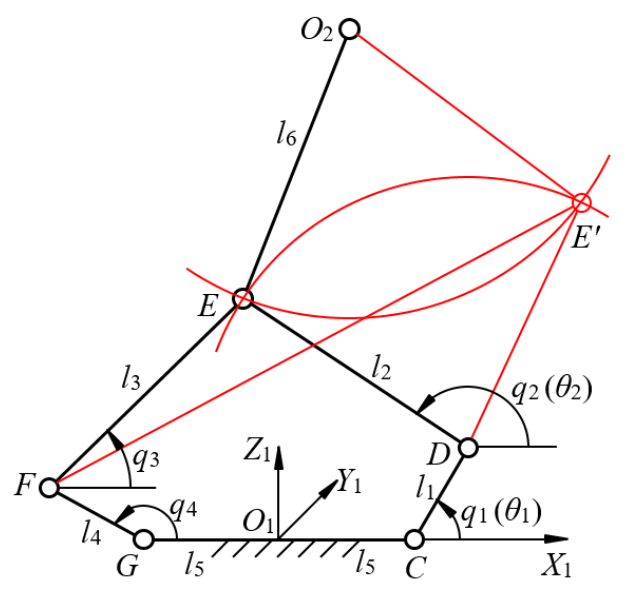

Fig. 2 HRDL

\section{Inverse and forward position analyses}

\subsection{The inverse position analysis of the 3-PSS/7R HRDM}

The inverse kinematics of the 3-PSS/7R HRDM is to solve the displacement $h_{i}(i=1,2,3)$ of driving slider $S_{i}$ in three PSS limbs and rotation angle of the servo motor for the given position and orientation $(x, y, z, \alpha, \beta, \gamma)$ of the moving platform. Under the condition that the velocity and initial posture of the $\mathrm{CV}$ motor are known, the position vector of point $O_{2}$ in the $\left\{O_{1}\right\}$ is $\boldsymbol{O}_{2}=\left[\begin{array}{lll}x & y & z\end{array}\right]^{\mathrm{T}}$. According to the dimensions of the mechanism and the geometrical relationship among the links, the hinge centre coordinate values in respective coordinate system of four driving limbs can be obtained using vector projection method.

\subsubsection{The inverse position analysis of HRDL}

The middle limb of the 3-PSS/7R HRDM consists of the five-bar mechanism $C D E F G$ and link $E O_{2}$, the degree of freedom of the centre of the moving platform is completely determined by the middle limb. Thus the motion transformation matrix of the middle limb directly determines the transformation matrix of the whole mechanism.

The middle limb can be seen as consist of two sublimbs $O_{1} C D E O_{2}$ and $O_{1} G F E O_{2}$. The two sub-limbs are recombined at point $E$, the revolute pairs of point $E$ are coaxial. The position and orientation of the moving platform are controlled by two different types of driving forces acting on point $E$. In order to facilitate analysis, one of the sub-limbs $\mathrm{O}_{1} \mathrm{CDEO}_{2}$ is analysed, and the link coordinate systems of the middle limb $7 \mathrm{R}$ are established, as shown in Fig. 3. D-H 
parameters $[28,29]$ are defined for the middle limb, and $\theta_{i}$ $(i=1,2,3,4)$ can be obtained as shown in Table 1 .

Table 1

The D-H parameters of the sub-limb $\mathrm{O}_{1} \mathrm{CDEO}_{2}$

\begin{tabular}{|c|c|c|c|c|}
\hline No. $i$ & Link length $a_{i}$ & Torsion angle $\alpha_{i-1}$ & Bias $d_{i}$ & Turning angle $\theta_{i}$ \\
\hline 0 & 0 & $90^{\circ}$ & 0 & $0^{\circ}$ \\
\hline 1 & $l_{5}$ & 0 & 0 & $\theta_{1}$ \\
\hline 2 & $l_{1}$ & 0 & 0 & $\theta_{2}$ \\
\hline 3 & $l_{6}$ & 0 & 0 & $\theta_{3}$ \\
\hline 4 & 0 & 0 & 0 & $\theta_{4}$ \\
\hline$O_{2}$ & 0 & $-90^{\circ}$ & 0 & $0^{\circ}$ \\
\hline
\end{tabular}

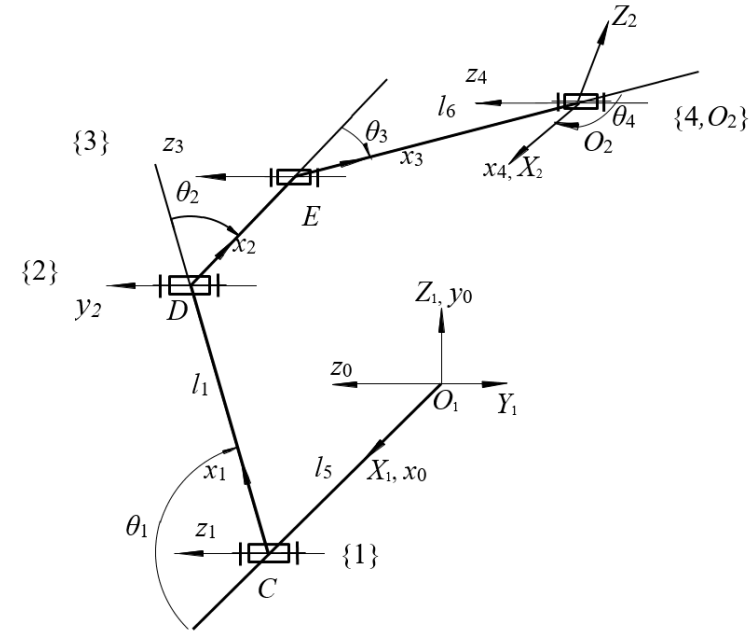

Fig. 3 The link coordinate systems of $\mathrm{O}_{1} \mathrm{CDEO}_{2}$

With D-H parameters, the general expression of the coordinate transformation matrix ${ }_{i-1}^{i} \boldsymbol{T}$ is as follows [30, $31]$.

$$
\begin{aligned}
& { }_{i}^{i-1} \boldsymbol{T}=\operatorname{Rot}\left(x, \alpha_{i-1}\right) \operatorname{Trans}\left(x, a_{i-1}\right) \operatorname{Trans}\left(z, d_{i}\right) \operatorname{Rot}\left(z, \theta_{i}\right)= \\
& =\left[\begin{array}{cccc}
\mathrm{c} \theta_{i} & -\mathrm{s} \theta_{i} & 0 & a_{i-1} \\
\mathrm{~s} \theta_{i} \mathrm{c} \alpha_{i-1} & \mathrm{c} \theta_{i} \mathrm{c} \alpha_{i-1} & -\mathrm{s} \alpha_{i-1} & -d_{i} \mathrm{~s} \alpha_{i-1} \\
\mathrm{~s} \theta_{i} s \alpha_{i-1} & \mathrm{c} \theta_{i} \mathrm{~s} \alpha_{i-1} & \mathrm{c} \alpha_{i-1} & d_{i} \mathrm{c} \alpha_{i-1} \\
0 & 0 & 0 & 1
\end{array}\right],
\end{aligned}
$$

$$
{ }_{O_{2}}^{O_{1}} \boldsymbol{T}={ }_{0}^{O_{1}} \boldsymbol{T}_{1}^{0} \boldsymbol{T}_{2}^{1} \boldsymbol{T}_{3}^{2} \boldsymbol{T}_{4}^{3} \boldsymbol{T}{ }_{O_{2}}^{4} \boldsymbol{T} .
$$

The orientation transformation matrix of the $\left\{\mathrm{O}_{2}\right\}$ relative to the $\left\{O_{1}\right\}$ is represented by the Euler angle as follows:

$$
{ }_{O_{2}}^{O_{1}} \boldsymbol{T}=\left[\begin{array}{cccc}
\mathrm{c} \beta \mathrm{c} \gamma & \mathrm{s} \alpha \mathrm{s} \beta \mathrm{c} \gamma-\mathrm{c} \alpha \mathrm{s} \gamma & \mathrm{c} \alpha \mathrm{s} \beta \mathrm{c} \gamma+\mathrm{s} \alpha \mathrm{s} \gamma & x \\
\mathrm{c} \beta \mathrm{s} \gamma & \mathrm{s} \alpha \mathrm{s} \beta \mathrm{s} \gamma+\mathrm{c} \alpha \mathrm{c} \gamma & \mathrm{c} \alpha \mathrm{s} \beta \mathrm{s} \gamma-\mathrm{s} \alpha \mathrm{c} \gamma & y \\
-\mathrm{s} \beta & \mathrm{s} \alpha \mathrm{c} \beta & \mathrm{c} \alpha \mathrm{c} \beta & z \\
0 & 0 & 0 & 1
\end{array}\right] \text {, }
$$

where: point $\boldsymbol{P}=\left[P_{x}, P_{y}, P_{z}\right]^{\mathrm{T}}=[x, y, z]^{\mathrm{T}}$ is the reference point and its vector coordinate is $\boldsymbol{P}=\left[\begin{array}{lll}x & y & z\end{array}\right]^{\mathrm{T}}$, which coincides with the coordinate origin of the moving coordinate system $\left\{\mathrm{O}_{2}\right\} .(\alpha, \beta, \gamma)$ are Euler angles of the frame $\left\{\mathrm{O}_{2}\right\}$.
The left side of Eq. (3) can be found by homogeneous transformation with D-H parameters. By comparing two sides of the equations, we have:

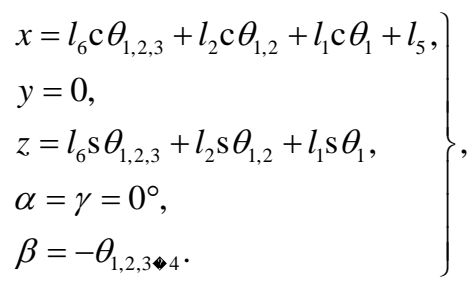

where: $\theta_{1,2, \cdots, k}=\theta_{1}+\theta_{2}+\cdots+\theta_{k}(k \in N)$,

$\mathrm{s} \theta_{1,2, \cdots, k}=\sin \left(\theta_{1}+\theta_{2}+\cdots+\theta_{k}\right)$,

$\mathrm{c} \theta_{1,2, \cdots, k}=\cos \left(\theta_{1}+\theta_{2}+\cdots+\theta_{k}\right), \mathrm{s}=\sin$ and $\mathrm{c}=\cos$.

Substituting Eq. (4) into Eq. (3), yields:

$$
{ }_{O_{2}}^{O_{1}} \boldsymbol{T}=\left[\begin{array}{cccc}
\mathrm{c} \beta & 0 & \mathrm{~s} \beta & x \\
0 & 1 & 0 & 0 \\
-\mathrm{s} \beta & 0 & \mathrm{c} \beta & z \\
0 & 0 & 0 & 1
\end{array}\right]
$$

When the parameters of the position and orientation of the moving platform $(x, z, \beta)^{\mathrm{T}}$ and parameters of the constraint limbs are determined, the value of the relative absolute coordinates of each joint is obtained through the form of multiple function products. The input parameters of two motors can be determined by means of the trajectory of point $E$ because point $E$ is driven by the servo motor and the $\mathrm{CV}$ motor.

The transformation matrix of the known limb as Eq. (2), the coordinate vector of point $E$ can be obtained by homogeneous transformation.

$$
{ }_{3}^{O_{1}} \boldsymbol{T}=\left({ }_{0}^{O_{1}} \boldsymbol{T}_{1}^{0} \boldsymbol{T}_{2}^{1} \boldsymbol{T}_{3}^{2} \boldsymbol{T}\right)={ }_{O_{2}}^{O_{1}} \boldsymbol{T}\left({ }_{4}^{3} \boldsymbol{T}{ }_{O_{2}}^{4} \boldsymbol{T}\right)^{-1} .
$$

From Eq. (6), the following expression can be obtained:

$$
\left.\begin{array}{l}
{ }^{3} p_{x}=x_{e}=x-l_{6} \mathrm{c}\left(\beta-\theta_{4}\right), \\
{ }^{3} p_{y}=y_{e}=0, \\
{ }^{3} p_{z}=z_{e}=z-l_{6} \mathrm{~s}\left(\beta-\theta_{4}\right)
\end{array}\right\},
$$

where: $\left(x_{e}, y_{e}, z_{e}\right)^{\mathrm{T}}$ is the coordinate of the point $E$ in the middle limb 7R.

When the position and orientation of moving platform $(x, z, \beta)^{\mathrm{T}}$ and the driving angle of the CV motor $\theta_{1}$ is determined, it's easy to get the coordinates of point $E$ in the $\left\{O_{1}\right\}$. From Fig. 2, the vector equations of limb $O_{1} C D E$ driven by the $\mathrm{CV}$ motor and limb $O_{1} G F D E$ driven by the servo motor are established, respectively, using closed vector method, as follows:

$$
\begin{aligned}
& O_{1} E=O_{1} C+C D+D E, \\
& O_{1} E=O_{1} G+G F+F E .
\end{aligned}
$$

Their projects to $x_{1}$ and $z_{1}$ axes yield: 


$$
\left.\begin{array}{l}
x_{e}=l_{5}+l_{1} \mathrm{c} q_{1}+l_{2} \mathrm{c} q_{1,2}, \\
z_{e}=l_{1} \mathrm{~s} q_{1}+l_{2} \mathrm{~s} q_{1,2} .
\end{array}\right\},
$$

Eliminating $q_{1,2}$ from Eq. (10), yield:

$$
\left(x_{e}-l_{5}\right)^{2}-2 l_{1}\left(x_{e}-l_{5}\right) \mathrm{c} q_{1}+z_{e}^{2}-2 l_{1} z_{e} \mathrm{~s} q_{1}+l_{1}^{2}=l_{2}^{2} \text {. }
$$

Eliminating $\left(\beta-\theta_{4}\right)$ from Eq. (7), yield:

$$
\left(x-x_{e}\right)^{2}+\left(z-z_{e}\right)^{2}=l_{6}^{2} \text {. }
$$

From Eq. (12) and Eq. (13), get:

$$
z_{e}=\frac{-2 l_{5} x_{e}+l_{5}^{2}-2 l_{1}\left(x_{e}-l_{5}\right) \mathrm{c} q_{1}+l_{1}^{2}-l_{2}^{2}-x^{2}+2 x x_{e}-z^{2}}{\left(2 l_{1} \mathrm{~s} q_{1}-2 z\right)} .
$$

Substituting Eq. (14) into Eq. (12), yield:

$$
\begin{aligned}
& x^{2}-2 x x_{e}+x_{e}^{2}+z^{2}-2 B z x_{e}-\frac{2 C z}{A}+ \\
& +\frac{1}{A^{2}}\left(B^{2} x_{e}^{2}+2 B C x_{e}+C\right)^{2}=l_{6}^{2},
\end{aligned}
$$

where: $A=2 l_{1} \sin q_{1}-2 z, B=2 x-2 l_{5}-2 l_{1} \cos q_{1}$, $C=l_{5}^{2}-2 l_{1} l_{5} \cos q_{1}+l_{1}^{2}-l_{2}^{2}-x^{2}-z^{2}$. follows:

Eq. (15) is rewritten as an expression about $x_{e}$ as

$$
C x_{e}^{2}-D x_{e}+E=0
$$

where: $C=1+B^{2} / A^{2}, \quad D=2 x+2 B z / A-2 B C / A^{2}$, $E=x^{2}+z^{2}-2 C z / A+C^{2} / A^{2}-l_{6}^{2}$.

Solving Eq. (16), and get:

$$
x_{e}=\left(D \pm \sqrt{D^{2}-4 C E}\right) /(2 C)
$$

Under the working condition, the posture $X$ and $q_{1}$ of the HRDM at any moment are determined. For the limb $\mathrm{O}_{1} C D E \mathrm{O}_{2}$ driven by the $\mathrm{CV}$ motor, the following vector relationship is satisfied:

$$
\begin{aligned}
& O_{1} E+E O_{2}=O_{1} O_{2}, \\
& O_{1} E=O_{1} C+C D+D E .
\end{aligned}
$$

Under this condition, the solution is obtained as point $E$ and point $E^{\prime}$, but point $E$ is the coupling point of two limbs $O_{1} C D E$ and $O_{1} G F E$, thus the following conditions should be met.

$$
O_{1} E=O_{1} G+G F+F E
$$

As can be seen from Fig. 4, if both point $E$ and $E^{\prime}$ can meet the requirements of the limb $O_{1} G F E$, then there must be $|\boldsymbol{F E}|=\left|\boldsymbol{F} \boldsymbol{E}^{\prime}\right|,|\boldsymbol{D E}|=\left|\boldsymbol{D E} \boldsymbol{E}^{\prime}\right|$.

At the time $\triangle \boldsymbol{F} \boldsymbol{E} \boldsymbol{D} \cong \triangle \boldsymbol{F} \boldsymbol{E}^{\prime} \boldsymbol{D}$.

Then get, $\angle \boldsymbol{E F D}=\Delta \boldsymbol{E}^{\prime} \boldsymbol{F D}, \angle \boldsymbol{E D F}=\Delta \boldsymbol{E}^{\prime} \boldsymbol{D} \boldsymbol{F}$, $\angle F E D=\Delta F E^{\prime} D$.

Thus, point $E$ and $E^{\prime}$ overlap. However, this contradicts the fact that point $E$ and $E^{\prime}$ are two different points, thus it can both satisfy the uniqueness of $E$ driven by two limbs at the same time. Set the coordinate vector $\boldsymbol{E}$ as $\boldsymbol{X}_{e}=\left[x_{e}, z_{e}\right]^{\mathrm{T}}, z_{e}$ is located above the $Z_{1}$ axis and satisfies the assembly relationship:

$$
\begin{aligned}
& |F G-E F| \leq\left|O_{1} E\right| \leq|F G+E F| \\
& |C D-D E| \leq\left|O_{1} E\right| \leq|C D+D E|
\end{aligned} .
$$

Then the inverse solution of the HRDL can be obtained:

$$
\left.\begin{array}{l}
q_{1}=\arctan \left(\frac{z_{e}}{x_{e}-l_{6}}\right)-\arctan \frac{l_{2} \sin \left(q_{2}\right)}{l_{1}+l_{2} \cos \left(q_{2}\right)}, \\
q_{2}=\arctan \frac{ \pm \sqrt{1-F^{2}}}{F}, \\
q_{3}=\arctan \frac{ \pm \sqrt{1-G^{2}}}{G}, \\
q_{4}=\arctan \left(\frac{z_{e}}{x_{e}+l_{6}}\right)-\arctan \frac{l_{3} \sin \left(q_{3}\right)}{l_{4}+l_{3} \cos \left(q_{3}\right)} .
\end{array}\right\},
$$

where: $F=\frac{\left(x_{e}-l_{6}\right)^{2}+z_{e}^{2}-l_{1}^{2}-l_{2}^{2}}{2 l_{1} l_{2}}$,

$$
G=\frac{\left(x_{e}+l_{6}\right)^{2}+z_{e}^{2}-l_{3}^{2}-l_{4}^{2}}{2 l_{3} l_{4}} \text {. }
$$

Eq. (22) is the inverse solution of the HRDL, and each has two solutions. Since link $C D$ is driven by the $C V$ motor and can only move in one direction, $q_{1}, q_{2}$ is a given value. The value $q_{4}$ is the servo motor compensation angle, there are two possibilities according to the actual situation. The value $q_{3}$ is the angle of transition from $l_{3}$ to $l_{4}$ can be determined according to the assembly relationship of the initial mechanism.

\subsubsection{The inverse position analysis of the limb 3-PSS}

The position vector of point $B_{j}(j=1,2,3)$ of the base in the $\left\{O_{1}\right\}$ is $\boldsymbol{B}_{\mathrm{j}}=\left[\begin{array}{lll}B_{j x} & B_{j y} & B_{j z}\end{array}\right]^{\mathrm{T}}$.

$$
\boldsymbol{B}_{j}=\operatorname{Rot}(Z,(2 j-1) \pi / 3)\left[\begin{array}{lll}
R & 0 & 0
\end{array}\right]^{\mathrm{T}} .
$$

The coordinates of the prismatic pair $S_{j}$ in the $\left\{O_{1}\right\}$ can be expressed as: 


$$
\boldsymbol{S}_{j}=\operatorname{Rot}(Z,(2 j-1) \pi / 3)\left[\begin{array}{lll}
R & 0 & h_{j}
\end{array}\right]^{\mathrm{T}}
$$

The position vector of the spherical joint centre $P_{j}$ of the moving platform in the $\left\{\mathrm{O}_{2}\right\}$ is ${ }^{O_{2}} \boldsymbol{P}_{j}$.

$$
{ }^{o_{2}} \boldsymbol{P}_{j}=\operatorname{Rot}(Z,(2 j-1) \pi / 3)\left[\begin{array}{lll}
r & 0 & 0
\end{array}\right]^{\mathrm{T}} .
$$

Any vector in the $\left\{\mathrm{O}_{2}\right\}$ can be transformed to the $\left\{O_{1}\right\}$ by the coordinate transformation equation ${ }_{O_{2}}^{O_{1}} \boldsymbol{R}$ :

$$
{ }^{O_{1}} \boldsymbol{P}_{j}={ }_{O_{2}}^{O_{1}} \boldsymbol{R}^{O_{2}} \boldsymbol{P}_{j}+\boldsymbol{P},
$$

where, ${ }_{O_{2}}^{O_{1}} \boldsymbol{R}$ is the orientation transformation matrix in the form of $Z-Y$ - $X$ Euler angle $(\gamma, \beta, \alpha)$.

According to the characteristics of the 3-PSS/7R mechanism, the moving platform has three DOFs (two translations and one rotation). The middle HRDL 7R restricts the two rotation DOFs and one translation DOF of the moving platform. Its motion transformation matrix can be obtained through D-H parameters transformation of the middle limb 7R in Section 3.1.1. Thus the orientation transformation matrix ${ }_{O_{2}}^{O_{1}} \boldsymbol{R}$ can be expressed as:

$$
{ }_{O_{2}}^{O_{1}} \boldsymbol{R}=\left[\begin{array}{ccc}
\mathrm{c} \beta & 0 & \mathrm{~s} \beta \\
0 & 1 & 0 \\
-\mathrm{s} \beta & 0 & \mathrm{c} \beta
\end{array}\right]
$$

When generalized coordinates of the moving platform are known and substituting it into Eq. (26), the displacement vector of the spherical joint centre $P_{j}$ of the moving platform in the $\left\{O_{1}\right\}$ can be obtained. The length of the driving link also can be obtained using geometric relationships.

$$
\boldsymbol{L}_{j}=\left|\boldsymbol{S}_{j} \boldsymbol{P}_{j}\right|=\left|\boldsymbol{O}_{1} \boldsymbol{S}_{j}-\boldsymbol{O}_{1} \boldsymbol{P}_{j}\right|
$$

From Eq. (28), the length of the three limbs can be obtained by using coordinates of the spherical joint centres in the $\left\{O_{1}\right\}$, which connect to the base and the moving platform.

$$
L_{j}^{2}=L_{j x}^{2}+L_{j y}^{2}+L_{j z}^{2},
$$

where: $L_{j x}=P_{j x}-S_{j x}, \quad L_{j y}=P_{j y}-S_{j y}, \quad L_{j z}=P_{j z}-S_{j z}$.

Because the moving platform is located up the base, and the $z$ value of the coordinate values $(x, y, z)$ of reference point $O_{2}$ is constant positive and $z>h_{j}$, here the moving distance $h_{j}$ of the prismatic pairs is positive value and can be expressed as:

$$
h_{j}=S_{j z}-\sqrt{L_{j}^{2}-L_{j x}^{2}-L_{j y}^{2}} .
$$

The inverse solution equation of the PSS limbs can be obtained by substituting the mechanism parameters into Eq. (30).

$$
\left.\begin{array}{l}
h_{1}=z-0.5 r \mathrm{~s} \beta-a, \\
h_{2}=z+r \mathrm{~s} \beta-b, \\
h_{3}=z-0.5 r \mathrm{~s} \beta-a .
\end{array}\right\},
$$

where: $a=\sqrt{L^{2}-\delta_{1}^{2}-0.75(R-r)^{2}}, b=\sqrt{L^{2}-\delta_{2}^{2}}$, $\delta_{1}=x+0.5 r \mathrm{c} \beta-0.5 R, \delta_{2}=x-r \mathrm{c} \beta+R$.

Eq. (31) indicates that the mechanism can realize controlled motion of the moving platform by using coordinate values of each spherical joint centre in the $\left\{O_{1}\right\}$.

\subsection{Jacobian matrix of the 3-PSS/7R HRDM}

The HRDM's Jacobian mapping matrix is a nonfull rank matrix. The mechanism has 5 motion inputs but only 3 motion outputs. When the feeding velocity of the reference point of the moving platform is given, only five driving input velocities can be obtained, and the expression can be written as follows:

$$
\dot{Q}=J \dot{X}
$$

where: $\dot{X}=(\dot{x}, \dot{z}, \dot{\beta})^{\mathrm{T}}$ is the generalized velocity vector of the centre of the moving platform; $\dot{Q}$ is the velocity vector driven by the generalized joint.

\subsubsection{The velocity analysis of the middle limb $7 \mathrm{R}$}

The velocity of point $E$ is formed by coupling $C D E$ limb driven by $\mathrm{CV}$ motor and $G F E$ limb driven by servo motor. The velocity mapping matrix of HRDL is determined by the parallel five-bar mechanism and the spatial linkage. The velocity Jacobian of HRDL is expressed as ${ }^{1} \boldsymbol{J}$. The velocity of the CV motor $\dot{q}_{1}$ and the servo motor $\dot{q}_{4}$ and the output velocity of the centre point $\mathrm{O}_{2}$ of the moving platform satisfy the following equation:

$$
\left(\dot{q}_{1}, \dot{q}_{4}\right)^{\mathrm{T}}={ }^{1} \boldsymbol{J} \dot{\boldsymbol{X}}
$$

Under the driving $q_{1}$ and $q_{4}$, the velocity mapping relation of point $E$ is:

$$
\left(\dot{q}_{1}, \dot{q}_{4}\right)^{\mathrm{T}}={ }_{1}^{1} \boldsymbol{J}\left(\dot{x}_{e}, \dot{z}_{e}\right)^{\mathrm{T}}
$$

where: ${ }_{1}^{1} \boldsymbol{J}=\mathrm{s}\left(q_{2}-q_{3}\right)$.

$$
\left[\begin{array}{cc}
-l_{1} \mathrm{~s} q_{1} \mathrm{~s}\left(q_{2}-q_{3}\right)+l_{1} \mathrm{~s} q_{2} \mathrm{~s}\left(q_{1}-q_{3}\right) & -l_{4} \mathrm{~s} q_{2} \mathrm{~s}\left(q_{4}-q_{3}\right) \\
l_{1} \mathrm{c} q_{1} \mathrm{~s}\left(q_{2}-q_{3}\right)-l_{1} \mathrm{c} q_{2} \mathrm{~s}\left(q_{1}-q_{3}\right) & l_{4} \mathrm{c} q_{2} \mathrm{~s}\left(q_{4}-q_{3}\right)
\end{array}\right]^{-1} .
$$

The velocity mapping relationship between the spatial link and the moving platform is:

$$
\left(\dot{x}_{e}, \dot{z}_{e}\right)^{\mathrm{T}}={ }_{2}^{1} \mathrm{~J}(\dot{x}, \dot{z}, \dot{\beta})^{\mathrm{T}} \text {. }
$$

According to Eq. (7), point $E$ is determined at any time when the HRDM is in working state. The virtual limb $O_{1} E O_{2}$ can be regarded as the passive link of the parallel mechanism with a middle passive link [21].

For the virtual limb $\mathrm{O}_{1} E O_{2}$, the motion mapping 
relation is equivalent to:

$$
J^{\prime} \dot{\theta}^{\prime}=\dot{X}
$$

where: $\dot{\boldsymbol{\theta}}^{\prime}=\left[\begin{array}{lll}\dot{\theta}_{1}{ }^{\prime} & \dot{\theta}_{2}{ }^{\prime} & \theta_{3}{ }^{\prime}\end{array}\right]^{\mathrm{T}}$ is the velocity vectors of $O_{1}, E$ and $\mathrm{O}_{2}$ of the virtual revolute joints. $\boldsymbol{J}^{\prime}$ is the Jacobian matrix of the virtual limb, which can be expressed as:

$$
\boldsymbol{J}^{\prime}=\left[\begin{array}{ccc}
\boldsymbol{e}_{1} & \cdots & \boldsymbol{e}_{3} \\
\boldsymbol{e}_{1} \times \boldsymbol{r}_{1}^{\prime} & \cdots & \boldsymbol{e}_{3} \times \boldsymbol{r}_{3}^{\prime}
\end{array}\right],
$$

where: $\boldsymbol{r}_{i}{ }^{\prime}$ is the position vector relative to the centre of the static platform, $\boldsymbol{r}_{2}^{\prime}=\boldsymbol{r}_{\mathrm{e}}^{\prime}, \theta_{3}^{\prime}=\theta_{4}$.

Thus,

$$
{ }^{1} \boldsymbol{J}={ }_{1}^{1} \boldsymbol{J} \cdot{ }_{2}^{1} \boldsymbol{J}
$$

\subsubsection{The velocity analysis of the driving limbs 3-PSS}

The velocity equation can be obtained by differentiating the position equation with respect to time. The position and orientation of the moving platform are constantly varying when the mechanism is in the working state. In order to obtain the driving velocities of three prismatic pairs of the limbs 3-PSS and the moving velocity of the moving platform, the velocity equation can be obtained by taking position equation to the first derivative with respect to time.

$$
\dot{\boldsymbol{q}}={ }^{2} \boldsymbol{J} \dot{\boldsymbol{X}}
$$

where: $\dot{\boldsymbol{q}}=\left(\dot{h}_{1}, \dot{h}_{2}, \dot{h}_{3}\right)^{\mathrm{T}}$ is driving velocity vector.

The Jacobian matrix of the 3-PSS limbs can be obtained by differentiating Eq. (31) with respect to time, yields.

$$
{ }^{2} \boldsymbol{J}=\left[\begin{array}{ccc}
\frac{\delta_{1}}{a} & 1 & -\frac{r\left(\mathrm{~s} \beta \delta_{1}+\mathrm{c} \beta a\right)}{2 a} \\
\frac{\delta_{2}}{b} & 1 & \frac{r\left(\mathrm{~s} \beta \delta_{2}+\mathrm{c} \beta b\right)}{b} \\
\frac{\delta_{1}}{c} & 1 & -\frac{r\left(\mathrm{~s} \beta \delta_{1}+\mathrm{c} \beta c\right)}{2 c}
\end{array}\right] .
$$

is:

The total Jacobian matrix of the 3-PSS/7R HRDM

$$
\boldsymbol{J}=\left[\begin{array}{c}
{ }^{1} \boldsymbol{J} \\
2 \\
{ }^{J}
\end{array}\right]
$$

\section{The workspace atlas of the 3-PSS/7R HRDM}

\subsection{Factors affecting workspace of the HRDM}

Workspace is one of the important indexes to measure the kinematic performance of a mechanism. The HRDM workspace is the active area that can be reached by centre of moving platform, which size and shape determine the active space of the mechanism. The HRDM consists of
3-PSS limbs and a middle HRDL, and its workspace is affected by all the limbs.

The main factors affecting the workspace of 3-PSS parallel mechanism as: the activity degree of the ball hinge pair, the length range of the links, and the interference between the links. The mathematical relationship expression is as follows:

$$
W_{R}=\left\{\begin{array}{lr}
-\sigma_{\max } \leq \sigma_{j} \leq \sigma_{\max }, & j=1,2,3 \\
L_{\text {min }} \leq L_{j} \leq L_{\text {max }}, & j=1,2,3, \\
d_{i, k} \geq d_{\text {min }}, i \neq k, & i, k=1,2,3
\end{array},\right.
$$

where: $\sigma_{j}$ is the activity degree of the $j$ th spherical pair; $\sigma_{\max }$ is the extreme value of ball hinge mobile space; $L_{i}$ is the length of the $i$ th drive link; $L_{\min }$ and $L_{\max }$ is the limit of the length of the driving link; $d_{i, k}$ is the distance between the limbs; $d_{\min }$ is the limit distance between the links. are as follows:

The factors that affect the workspace of the HRDL

1) The parameters of the hybrid drive planar fivebar mechanism.

The workspace of 3-PSS mechanism is determined, and HRDL plays a constraint role in the whole mechanism, thus it determines the whole workspace of the mechanism. A key factor in the calculation of the HRDL workspace is to determine the workspace of the planar five-bar mechanism $C D E F G$. The shape of the workspace of point $E$ is determined by the two limbs $C D E$ and $E F G$. The length of the link and the frame of the planar five-bar mechanism also determine the workspace of point $E$. The constraint condition for calculating the workspace point set of point $E$ of the hybrid driven planar five-bar mechanism is:

$$
\left\{\begin{array}{l}
\left|l_{1}-l_{2}\right|<l_{e}<l_{1}+l_{2} \\
\left|l_{3}-l_{4}\right|<l_{e}<l_{3}+l_{4}
\end{array} .\right.
$$

2) The constraint of the connecting link length $l_{6}$.

The upper and lower limits of the trajectory of the moving platform centre are within the maximum and minimum driving range of 3-PSS sliding pair, respectively. $h_{\text {min }}$, $h_{\max }$ are the minimum and maximum stroke of 3-PSS sliding pairs, respectively. Then, the maximum motion region in $z$ direction of the 3-PSS mechanism is:

$$
\left\{\begin{array}{l}
z_{\min }^{\prime}=\sqrt{L_{i}^{2}-(R-r)^{2}}+h_{\min } \\
z_{\max }^{\prime}=\sqrt{L_{i}^{2}-(R-r)^{2}}+h_{\max }
\end{array} \quad(i=1,2,3) .\right.
$$

The workspaces of the HRDL is:

$$
\Omega \in\left\{\begin{array}{l}
\left|l_{1}-l_{2}\right|+l_{6}<l_{e}+l_{6}<l_{1}+l_{2}+l_{6} \\
\left|l_{3}-l_{4}\right|+l_{6}<l_{e}+l_{6}<l_{3}+l_{4}+l_{6}
\end{array} .\right.
$$

If there is an intersection between the HRDL and 3-PSS limbs, the following conditions should be satisfied.

$$
z_{\min }^{\prime} \leq \Omega_{z} \leq z_{\max }^{\prime}
$$

Since the degree of freedom of the whole mechanism is determined by the middle HRDL, the activity degree of the HRDL directly determines the activity ability of the entire mechanism. The mechanism has no linear movement 
ability in $y$ direction, and the workspace of the mechanism determined by the orientation and the parameters of each limb of the mechanism.

\subsection{Workspace atlas of the 3-PSS/7R HRDM}

The workspace of the 3-PSS/7R HRDM is the intersection of the two workspaces determined by the limbs 3 PSS and the middle HRDL 7R, respectively.

As shown in Fig. 1, assuming the moving distance $h_{i}$ of the driving slider $S_{i}(i=1,2,3)$ of the limbs 3-PSS is defined in the range of $[0 \mathrm{~mm}, 400 \mathrm{~mm}$ ]. The structure parameters of the 3-PSS/7R HRDM are shown in Table 2.

Table 2

The structure parameters of the 3-PSS/7R HRDM

\begin{tabular}{|l|c|c|}
\hline \multicolumn{1}{|c|}{ Description } & Parameters & Value \\
\hline $\begin{array}{l}\text { Lengths of the driving links in the } \\
\text { middle limb 7R }\end{array}$ & $l_{i}(i=1,4) / \mathrm{mm}$ & 120 \\
\hline $\begin{array}{l}\text { Lengths of the connecting links in the } \\
\text { middle limb 7R }\end{array}$ & $l_{i}(i=2,3) / \mathrm{mm}$ & 450 \\
\hline $\begin{array}{l}\text { Length of the frame in the middle } \\
\text { limb 7R }\end{array}$ & $215 / \mathrm{mm}$ & 600 \\
\hline $\begin{array}{l}\text { Length of link } E O_{2} \text { in the middle limb } \\
7 \mathrm{R}\end{array}$ & $16 / \mathrm{mm}$ & 420 \\
\hline $\begin{array}{l}\text { Maximum oscillating angle of the } \\
\text { spherical pair S }\end{array}$ & $\psi /\left(^{\circ}\right)$ & 45 \\
\hline Radius of the base & $R / \mathrm{mm}$ & 400 \\
\hline Radius of the moving platform & $r / \mathrm{mm}$ & 180 \\
\hline Length of link $P_{i} S_{i}$ & $L_{i}(i=1,2,3) / \mathrm{mm}$ & 500 \\
\hline
\end{tabular}

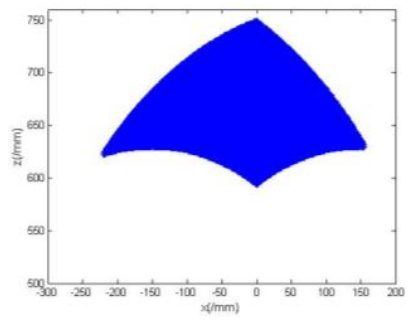

a) $\beta=0^{\circ}$

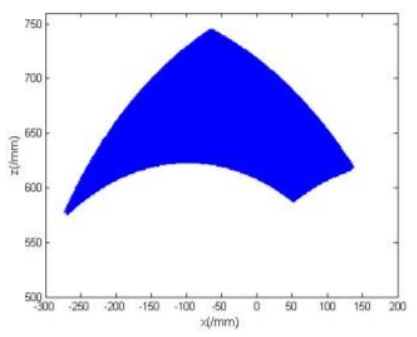

c) $\beta=10^{\circ}$

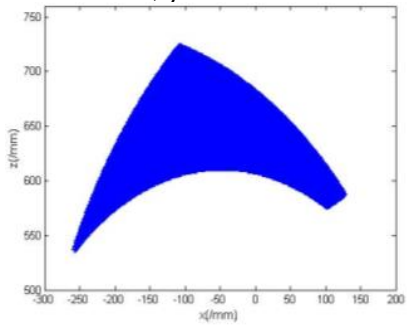

e) $\beta=20$

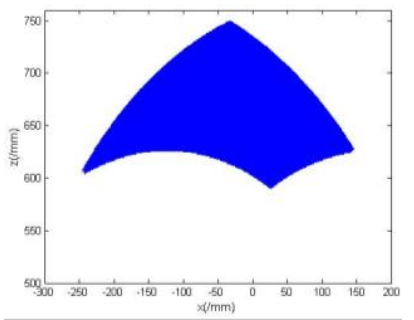

b) $\beta=5^{\circ}$

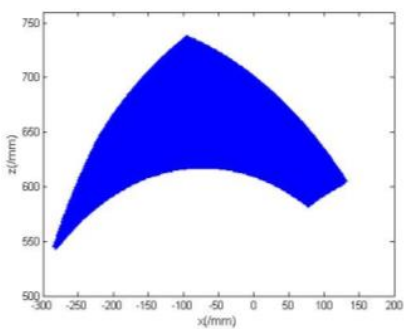

d) $\beta=15^{\circ}$

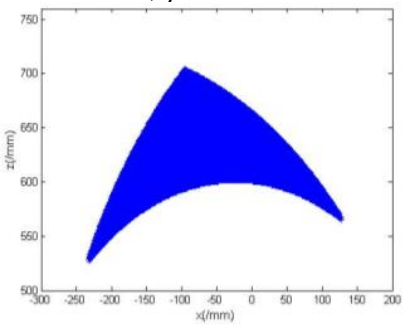

f) $\beta=25^{\circ}$
Fig. 4 Workspace atlas of the 3-PSS/7R HRDM with different orientation angles
The range of the flexible orientation angle $\beta$ is $\left[0^{\circ}\right.$, $\left.25^{\circ}\right]$. The constraint conditions of the workspace of the middle limb 7R are added to the workspace search program of the limbs 3-PSS, and the workspaces with different flexible orientation angles $\beta$ are obtained, as shown in Fig. 4.

From Fig. 4, the workspace of the 3-PSS/7R HRDM has the following characteristics:

1) As shown in Fig. 4, a, when the feasible orientation angle $\beta=0^{\circ}$, the workspace area is the largest. However, the minimum reachable space of the $z$ axis is less then when $\beta$ become larger.

2) When the flexible orientation angle $\beta$ increases gradually, the maximum working radius of the mechanism has no change, which is $[-250 \mathrm{~mm} 150 \mathrm{~mm}]$. However, there are many unreachable spaces in the working radius along $Z_{1}$ axis in the $\left\{O_{1}\right\}$, and the workspace area decreases obviously and the shape also changes.

\section{Case study of the 3-PSS/7R HRDM}

In order to verify the correctness of the kinematics models of the 3-PSS/7R HRDM, assuming the centre $\mathrm{O}_{2}$ of the moving platform moves along the linear motion trajectory in the workspace range, the linear motion law is:

$$
\left\{\begin{array}{l}
x=20 \sin (0.1 \cdot t) \\
y=\alpha=\beta=\gamma=0 \\
z=20 \cos (0.1 \cdot t)
\end{array} \quad(0 \leq t \leq 20) .\right.
$$

Assuming the angular velocity of the $\mathrm{CV}$ motor is $\dot{\theta}_{1}=18 \mathrm{deg} / \mathrm{s}$, and the initial point of $\theta_{10}=0$. The reference point of the moving platform moves in a uniform linear motion, its starting point $P_{0}$ is $(0,0,720)$ and the end point $P_{e}$ is $(100,0,670)$. According to the inverse kinematics model of the 3-PSS/7R HRDM established by the inverse solution Eqs. (22), (30), (38) and (41), the theoretical curves of the inverse kinematics solution of the mechanism are obtained, as shown in Figs. 5 and 6.

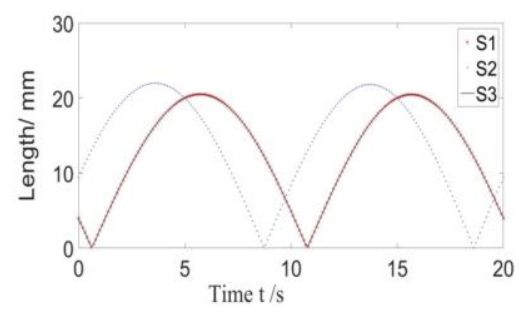

a) The displacements of the driving sliders $S_{i}(i=1,2,3)$

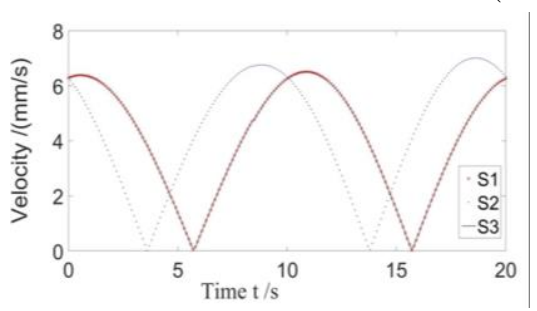

b) The velocities of the driving sliders $S_{i}(i=1,2,3)$

Fig. 5 The theoretical curves of the displacements and velocities of the driving sliders $S_{i}(i=1,2,3)$ 


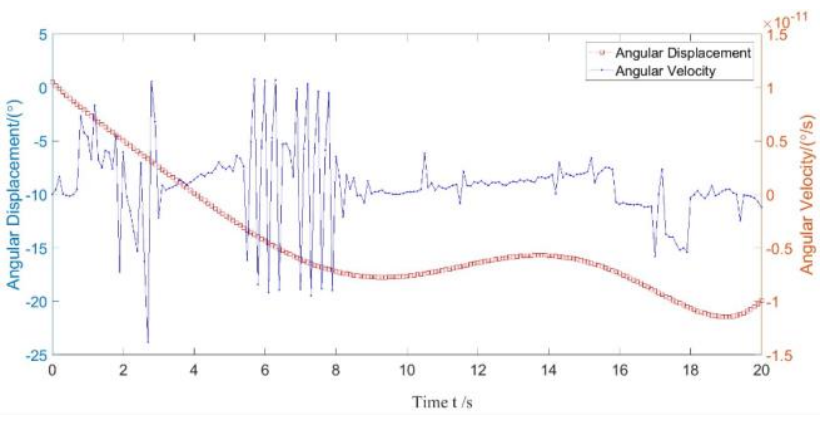

Fig. 6 The theoretical curves of angular displacement and angular velocity of the servo motor

As can be seen from Figs. 5 and 6, when the reference point of the moving platform moves along the straight line at a constant velocity, the maximum displacement of the drivers is within the range of its motion limit, the maximum movement velocity of the drivers is within the rated velocity of the motor, the acceleration changes gently, which is beneficial to the movement control of the 3-PSS/7R HRDM. One of the drivers of $7 \mathrm{R}$ limb is constant velocity, and the velocity and acceleration do not vary with time. The angular displacement of the servo motor is the cosine function, and the variation range of the angular velocity is from $0 \% \mathrm{~s}$ to $7.2 \%$, the variation range of the angular acceleration is from $30 \% \mathrm{~s}^{2}$ to $90 \% \mathrm{~s}^{2}$. The variation ranges of the angular velocity, angular acceleration, and the interval are narrow. The gentle change has little impact on the motor and mechanism, thus it is controllable.

\section{Conclusions}

A novel 3-PSS/7R HRDM with 2T1R motion mode is presented. The inverse position solution model of the mechanism is established. The $5 \times 3$ Jacobian matrix equation between the velocity of the moving platform and the driving velocity of the joint, which is suitable for Euler angular velocity, is established. The one-to-one correspondence between the joint space and the workspace velocity is realized.

The factors that affect the workspace of the mechanism are analysed, and the workspace atlas under different orientation angles are obtained.

The case simulation analysis results verify the rationality mechanism design, thus theoretically verify the feasibility of the HRDM realization and mechanism controllability, and lay the foundation for further trajectory precision control test research.

This study has been conducted only for 3-DOF mechanism and can serve as the basis for the kinematics of $n$-DOF $(n \geqslant 4)$ mechanisms.

The mechanism's research broadens the configuration of hybrid driven mechanisms to the space field, broadens the research scope of hybrid driven mechanisms, and provides a theoretical reference for the research of spatialization of hybrid driven mechanisms.

\section{Acknowledgements}

The work was financially supported by the Key Research and Development Program of Shanxi Province of China (International Cooperation, 201803D421028, 201903D421051).

\section{References}

1. Tokuz, L. C. 1992. Hybrid Machine Modelling and Control, Ph.D. thesis, Liverpool Polytechnic, UK.

2. Li, R. Q.; Wang, Y.; Wang, M. Y.; Zhao, Y. H.; Song, J. 2016. Research progress and development trend of hybrid-driven mechanism, Journal of Mechanical Engineering 52(13): 1-9 (in Chinese). https://doi.org/10.3901/JME.2016.13.001.

3. Dülger, L. C.; Kireçci, A.; Topalbekroglu, M. 2003. Modeling and simulation of a hybrid actuator, Mechanism and Machine Theory 38: 395-407. https://doi.org/10.1016/S0094-114X(02)00129-5.

4. Kütük, M. E.; Halicioglu, R.; Dulger, L. C. 2015. Kinematics and simulation of a hybrid mechanism: Matlab/SimMechanics, Journal of Physics: Conference Series 574: 012016_1-6. https://doi.org/10.1088/1742-6596/574/1/012016.

5. Kütük, M. E.; Dülger, L. C. 2016. A hybrid press system: motion design and inverse kinematics issues, Engineering Science and Technology, an International Journal 19: 846-856.

https://doi.org/10.1016/j.jestch.2015.11.012.

6. Kireçci, A.; Dulger, L. C. 2000. A study on a hybrid actuator, Mechanism and Machine Theory 35: 11411149.

https://doi.org/10.1016/S0094-114X(99)00059-2.

7. Gong, J.; Wang, X.; Huang, F; Zhang, Y. 2015. Dynamic performances analysis of hybrid press based on dependent generalized coordinates, Proceedings of the Institution of Mechanical Engineers, Part C: Journal of Mechanical Engineering Science 229(12): 2187-2194. https://doi.org/10.1177/0954406214557342.

8. Dang, X. Z.; Zhou, L. S.;Liang, D. .(2013). Dynamic performance analysis and simulation of hybrid-driven seven-bar mechanical press with double cranks, Advanced Materials Research 834-836: 1327-1332. https://doi.org/10.4028/www.scientific.net/AMR.834836.1327.

9. Wang, Z. R.; Dong, E. B.; Xu, M.; Yang, J. 2015. Circling turning locomotion of a new multiple closedchain-legs robot with hybrid-driven mechanism, Advanced Robotics 29(24): 1637-1648. https://doi.org/10.1080/01691864.2015.1071682.

10. Li, C. H.; Tso, P. L. 2008. Experimental study on a hybrid-driven servo press using iterative learning control, International Journal of Machine Tools and Manufacture 48: 209-219. https://doi.org/10.1016/j.ijmachtools.2007.08.014.

11. Li, H.; Zhang, Y. P. 2010. Seven-bar mechanical press with hybrid-driven mechanism for deep drawing; Part 1: kinematics analysis and optimum design, Journal of Mechanical Science and Technology, 24(11): 2153-2160. https://doi.org/10.1007/s12206-010-0819-0.

12. Chen, Z. H.; Wang Y.; Li, Y. 2007. Neuro-PID control of hybrid machines with 2-DOF for trajectory tracking problems, Proceedings of the IEEE International Conference on Automation and Logistics, Jinan, China, August 18-21. https://doi.org/10.1109/ICAL.2007.4338992.

13. Li, H.; Xue, Z. Q. 2008. Modeling and PID control of the hybrid-driven mechanical press, Proceedings of the IEEE 7th World Congress on Intelligent Control and $\mathrm{Au}-$ tomation, Chongqing, China, June 25-27. 
https://doi.org/10.1109/WCICA.2008.4594520.

14. Tso, P. L. 2010. Optimal design of a hybrid-driven servo press and experimental verification, ASME Journal of Mechanical Design 132: 034503_1-4. https://doi.org/10.1115/1.4000213.

15. He, K.; Luo, Y. X.; Kong, C. T.; Du, R. 2009. Trajectory planning, optimization and control of a hybrid mechanical press, WSEAS Transactions on Systems 8(5): 614-627. https://www.researchgate.net/publication/228527037.

16. Zi, B.; Ding, H. F.; Cao, J. B.; Zhu, Z. C.; Kecskeméthy, A. 2014. Integrated mechanism design and control for completely restrained hybrid-driven based cable parallel manipulators, Journal of Intelligent and Robotic Systems 74: 643-661.

https://doi.org/10.1007/s10846-013-9848-0.

17. Zi, B.; Cao, J. B.; Qian, S. 2014. Iterative learning tracking control of a hybrid-driven based three-cable parallel manipulator, Advances in Mechanical Engineering, 248385_1-15. https://doi.org/10.1155/2014/248385.

18. Zi, B.; Cao, J. B.; Zhu, Z. C.; Mitrouchev, P. 2013. Design, dynamics, and workspace of a hybrid-drivenbased cable parallel manipulator, Mathematical Problems in Engineering 914653_1-15. https://doi.org/10.1155/2013/914653.

19. Sun, H. H.; Zi, B.; Qian, S. 2013.Modeling and control of winding hybrid-driven based cable-parallel manipulator, Applied Mechanics and Materials 373-375: 111115.

https://doi.org/10.4028/www.scientific.net/AMM.373375.111

20. Zhang, D.; Gosselin C. M. 2001. Kinetostatic modeling of $n$-DOF parallel mechanisms with a passive constraining leg and prismatic actuators, ASME Journal of Mechanical Design 123: 375-381. https://doi.org/10.1093/aob/mcg206.

21. Zhang, D.; Gosselin, C. M. 2002. Kinetostatic modeling of parallel mechanisms with a passive constraining leg and revolute actuators, Mechanism and Machine Theory 37: 599-617. https://doi.org/10.1016/S0094-114X(02)00011-3.

22. Piccin, O.; Bayle, B.; Maurin, B.; Mathelin, M. D. 2009. Kinematic modeling of a 5-DOF parallel mechanism for semi-spherical workspace, Mechanism and Machine Theory 44: 1485-1496.

https://doi.org/10.1016/j.mechmachtheory.2008.11.012.

23. Gao, F.; Peng, B. B.; Zhao, H.; Li, W. M. 2006. A novel 5-DOF fully parallel kinematic machine tool, International Journal of Advanced Manufacturing Technology 31: 201-207.

https://doi.org/10.1007/s00170-005-0171-1.

24. He, J. F.; Jiang, H. Z.; Tong, Z. Z.; Li, B. P.; Han, J. W. 2012. Study on dynamic isotropy of a class of symmetric spatial parallel mechanisms with actuation redundancy, Journal of Vibration and Control 18(8): 11561164. https://doi.org/10.1177/1077546311409608.

25. Zhou, X.; Xu, Y. D.; Yao, J. T.; Zheng, K. J.; Zhao, Y. S. 2016. Stiffness modelling and comparison of the
5-UPS/PRPU parallel machine tool with its non-redundant counterpart, Proceedings of the Institution of Mechanical Engineers, Part B: Journal of Engineering Manufacture 231(9): 1646-1657.

https://doi.org/10.1177/0954405415600014.

26. Liu, S. W; Peng, G. L.; Gao, H. J. 2019. Dynamic modeling and terminal sliding mode control of a 3-DOF redundantly actuated parallel platform, Mechatronics 60(C): 26-33.

https://doi.org/10.1016/j.mechatronics.2019.04.001.

27. Lu, Y.; Dai, Z. H. 2016. Dynamics model of redundant hybrid manipulators connected in series by three or more different parallel manipulators with linear active legs, Mechanism and Machine Theory 103: 222-235. https://doi.org/10.1016/j.mechmachtheory.2016.05.003.

28. Wu, J.; Zhang, B. B.; Wang, L. P. 2016. Optimum design and performance comparison of a redundantly actuated solar tracker and its nonredundant counterpart, Solar Energy 127: 36-47. https://doi.org/10.1016/j.solener.2016.01.017.

29. Wen, S. H.; Qin, G. Q.; Zhang, B. W.; Lam, H. K.; Zhao, Y. S.; Wang, H. B. 2016. The study of model predictive control algorithm based on the force/position control scheme of the 5-DOF redundant actuation parallel robot, Robotics and Autonomous Systems 79: 12-25. https://doi.org/10.1016/j.robot.2016.02.002.

30. Craig, J. J. 1986. Introduction to robotics: Mechanics and control, Pearson Education, Inc.

31. Cai, Z. X.; Xie, B. 2015. Robotics (3th ed.), Beijing, China: Tsinghua University Press. (in Chinese)

\section{Qisheng ZHANG, Ruiqin LI, Jingjing LIANG}

\section{KINEMATICS AND WORKSPACE ATLAS OF 3-PSS/7R HYBRID REDUNDANTLY DRIVEN MECHANISM}

S u m m a r y

Aiming at the problem that the traditional hybriddriven mechanism is limited to planar mechanism, the paper presents a 3-PSS/7R Hybrid and Redundantly Driven Mechanism (HRDM for short), which takes advantage of hybrid driven and redundantly driven mechanism, driven by both constant velocity motor and servo motor. The mechanism consists of three PSS limbs with 6-DOF and a middle constrained limb with $7 \mathrm{R}$ configuration. The moving platform is constrained to 3-DOF motion. The kinematics model of the mechanism is established, and the kinematics analysis of 3-PSS/7R mechanism is carried out. The Jacobian matrix of the inverse kinematics is derived. The factors that affect the workspace are analyzed. The workspace of the HRDM is solved and verified using numerical method, and the workspace atlas with different orientation angles is obtained.

Keywords: 3-PSS/7R, hybrid redundantly driven mechanism (HRDM), Jacobian matrix, kinematics, workspace.

Received April 25, 2021

Accepted February 04, 2022 